\title{
Auditory noise influences human visual perception of ambiguous information: multi- modal integration during bistable perception
}

\author{
Woochul Choi", Se-Bum Paik \\ From 24th Annual Computational Neuroscience Meeting: CNS*2015 \\ Prague, Czech Republic. 18-23 July 2015
}

When the sensory system receives an ambiguous signal, human perception often switches spontaneously between two different interpretations. This phenomenon is called bistable perception, and has been considered important to understanding sensory system. In this study, we investigated the intervals of spontaneous switching, defined as reversal time $\tau$, to examine the temporal dynamics of bistable perception. We also studied the multi-modal feature of bistable switching by applying auditory noise with the visual stimuli. Our hypothesis is that auditory noise would significantly alter the reversal time of bistable visual perception. By building a computational model, we could explain the influence of auditory noise on the reversal time.

In the human psychophysical experiments, we first measured the reversal time with visual stimulus only, using two types of bistable visual movies: the racetrack [1] and the rotating 3D cylinder (Figure 1A). We observed that the reversal times are widely varied across the subjects but fairly consistent within the subject in both cases. Interestingly, we also found that the reversal time for the racetrack and the rotating cylinder were highly correlated $\left(N=9, R^{2}\right.$ $=0.84$, Figure $1 \mathrm{C}$ ). Next, we performed the experiment with auditory noise and visual stimuli together, and found that the reversal times are significantly altered. Importantly, when auditory noise was given, we found a systematic change such that a fast switching subjects (short $\tau$ ) tend to slow down the switching while slow switching subjects (long $\tau$ ) tend to speed up, so that the difference of $\tau$ between the two groups become insignificant (Figure 1D). Lastly, we designed a double-well energy model with

\footnotetext{
* Correspondence: choiwc1128@kaist.ac.kr

Department of Bio and Brain Engineering, KAIST, Daejeon 305-338, Republic
} of Korea

\section{and take full advantage of:}

- Convenient online submission

- Thorough peer review

- No space constraints or color figure charges

- Immediate publication on acceptance

- Inclusion in PubMed, CAS, Scopus and Google Scholar

- Research which is freely available for redistribution

Submit your manuscript at

C Biomed Central 


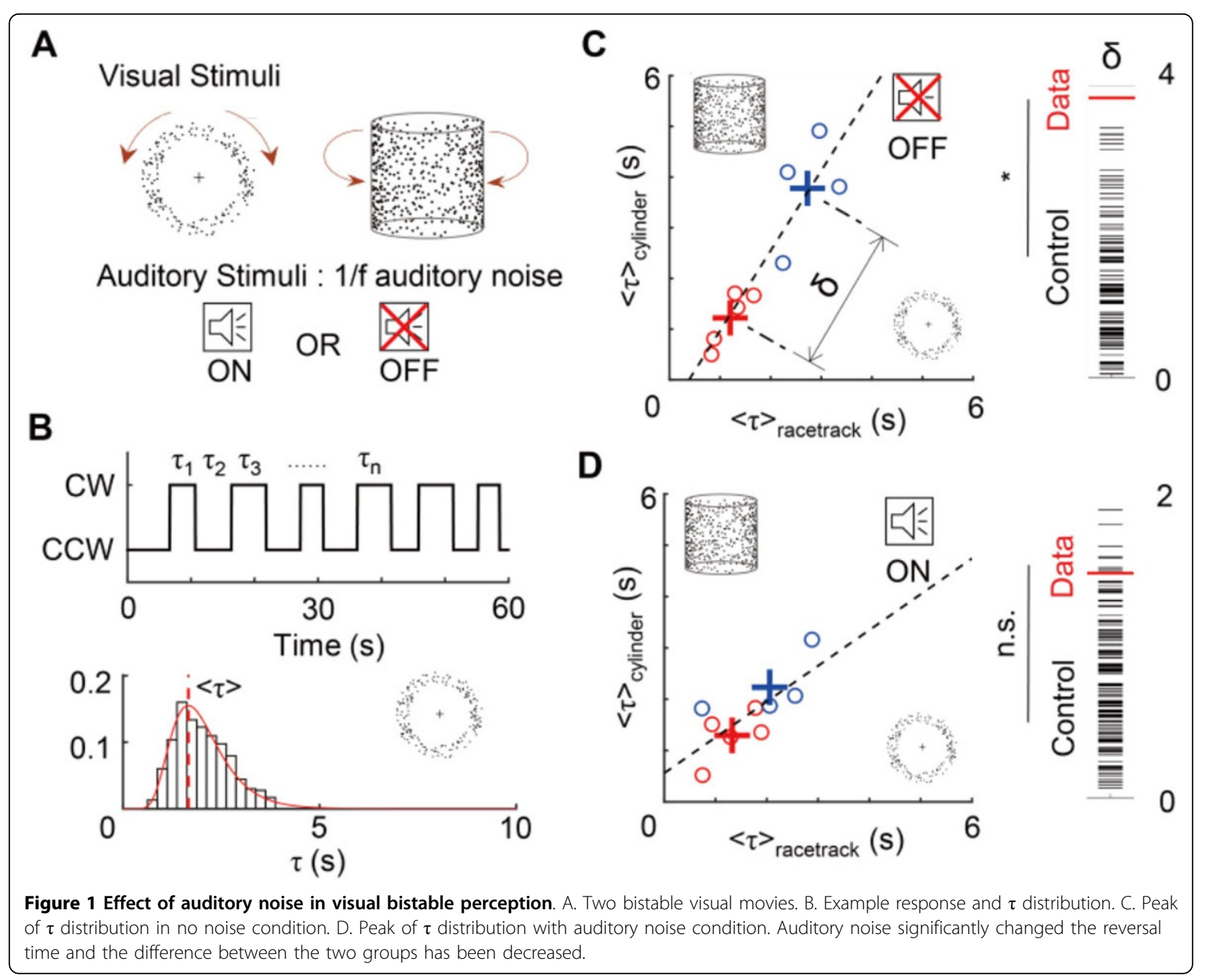

